\title{
Psychometric properties and longitudinal validation of the self-reporting questionnaire (SRQ-20) in a Rwandan community setting: a validation study
}

Willem F Scholte ${ }^{1,2^{*}}$, Femke Verduin ${ }^{1,2}$, Anouk van Lammeren ${ }^{1,2}$, Theoneste Rutayisire ${ }^{3}$ and Astrid M Kamperman ${ }^{1,2,4}$

\begin{abstract}
Background: This study took place to enable the measurement of the effects on mental health of a psychosocial intervention in Rwanda. It aimed to establish the capacities of the Self-Reporting Questionnaire (SRQ-20) to screen for mental disorder and to assess symptom change over time in a Rwandan community setting.

Methods: The SRQ-20 was translated into Kinyarwanda in a process of forward and back-translation. SRQ-20 data were collected in a Rwandan setting on 418 respondents; a random subsample of 230 respondents was assessed a second time with a three month time interval. Internal reliability was tested using Cronbach's alpha. The optimal cut-off point was determined by calculating Receiver Operating Curves, using semi-structured clinical interviews as standard in a random subsample of 99 respondents. Subsequently, predictive value, likelihood ratio, and interrater agreement were calculated. The factor structure of the SRQ-20 was determined through exploratory factor analysis. Factorial invariance over time was tested in a multigroup confirmatory factor analysis.
\end{abstract}

Results: The reliability of the SRQ-20 in women $(\alpha=0.85)$ and men $(\alpha=0.81)$ could be considered good. The instrument performed moderately well in detecting common mental disorders, with an area under the curve (AUC) of 0.76 for women and 0.74 for men. Cut-off scores were different for women (10) and men (8). Factor analysis yielded five factors, explaining 38\% of the total variance. The factor structure proved to be time invariant.

Conclusions: The SRQ-20 can be used as a screener to detect mental disorder in a Rwandan community setting, but cut-off scores need to be adjusted for women and men separately. The instrument also shows longitudinal factorial invariance, which is an important prerequisite for assessing changes in symptom severity. This is a significant finding as in non-western post-conflict settings the relevance of diagnostic categories is questionable. The use of the SRQ-20 can be considered an alternative option for measuring the effect of a psychosocial intervention on mental health.

Trial registration: Nederlands Trial Register NTR1120.

\section{Background}

A psychosocial intervention in Rwanda

The country of Rwanda experienced extreme violence during a genocidal three months period starting in April 1994. Over the preceding years, the country's northern

\footnotetext{
* Correspondence: w.f.scholte@amc.uva.nl

'Department of Psychiatry, Academic Medical Center, University of

Amsterdam, Netherlands, Meibergdreef 5, 1105 AZ Amsterdam, Netherlands Full list of author information is available at the end of the article
}

Gicumbi district had already been confronted with repeated acts of violence stemming from the same ethnic conflict. Although sixteen years have passed since, many inhabitants still suffer from the emotional sequelae.

Since early 2006 a psychosocial community intervention is carried out in the Northern Province in Rwanda, in and around a small city called Byumba. It is a therapeutic group intervention (sociotherapy) aiming at social 
bonding and mental health recovery [1-3]. No diagnostic criteria for participation have been defined, and the intervention is open to any adult ( $\geq 16$ years) wanting to participate. Community members can also personally be invited when considered psychosocial problem cases by local sociotherapy group facilitators. Under the lead of these facilitators, sociotherapy groups meet weekly during a period of fifteen weeks. Every four months a new series of groups start. Groups contain adult participants of both sexes with a wide age distribution.

\section{Measurement of mental health in a post-conflict setting}

When contemplating our choice for an instrument to measure the intervention's effect on mental health, we were aware of the questionnable relevance of diagnostic categories as defined by DSM-IV [4] or ICD-10 [5] in a population recently affected by systematic violence [6-8]. The high prevalence estimates of specific mental health problems usually established in such populations may reflect normal responses to severe environmental stress instead of disorders $[9,10]$. Additionally, the intervention studied does not focus on subjects suffering from any specific disorder. Instead, local community leaders' lay criteria for being a psychosocial problem case determine who will be beneficiaries of the intervention. Therefore, we chose to use a general case finding instrument rather than one or more instruments indicative of specific diagnoses. For this purpose we selected the Self-Reporting Questionnaire (SQR-20) [11,12].

\section{Validity of instrument translation}

As the SRQ-20 had never been used in Rwanda before, we needed to translate the instrument into the country's local language, Kinyarwanda, and to establish its validity and optimal cut-off point. English-language research instruments must be carefully adapted and translated before use in another culture. Different terminologies exist to classify criteria such adaptations must meet [13]. Manson [14] discusses adaptation of instrument items in terms of comprehensibility (meaning of item is evident), acceptability (item is not offensive), relevance (item relates to the underlying construct) and completeness (item fully covers equivalents between cultures). When connecting these terms to the forms of equivalence between original and translated instruments as mentioned earlier by Flaherty et al. [15], comprehensibility relates to semantic equivalence, acceptability to technical equivalence, relevance to content equivalence, and completeness to semantic, criterion or conceptual equivalence [13].

\section{Longitudinal validity}

We did not only use the SRQ-20 for its original purpose, i.e. case detection, but also to assess changes in scores over time. To our knowledge such use of the instrument has only been described once [16], but no data were provided about its longitudinal validity. We had to establish the instrument's capacity to meet additional psychometric criteria regarding its factorial solution. Changes in the score of a symptom checklist preferably reflect changes in the severity of the (possible) disorder. However, changes in test score may also reflect a reappraisal of the items, i.e. a reappraisal of the symptoms or a reappraisal of their impact. This makes changes in mean scores difficult to interpret. If the factorial solution of the instrument is stable over time, the latter sort of change can be ruled out since item loadings are affected by a reappraisal of the items [17]. Consequently, factorial invariance of the SRQ-20 is a prerequisite for assessing changes in symptom severity.

\section{Study objectives}

The aim of this study was multiple. First we assessed the SRQ-20's capacity to screen for mental disorder in a Rwandan community setting. Next, we evaluated the psychometric properties of the instrument. Finally, we tested the stability of the factorial solution over time.

\section{Methods}

\section{Ethics statement}

Approval for this study was gained from the Medical Ethics Committee of the Academic Medical Center in Amsterdam, Netherlands.

\section{Study site and population}

The sample for this study includes a mixture of beneficiaries of the intervention and their relatives, friends or close collaborators as well as individuals who were randomly selected in a nearby region not reached by the intervention.

\section{Instrument}

The SQR-20 could be expected to show reliability and validity for case detection in the Rwandan context. The instrument is a twenty items subset of the SRQ developed by the World Health Organization for screening the presence of mental disorder in patients contacting primary health care settings $[11,18]$. The complete SRQ consists of twenty-five questions, which have to be answered by 'yes' or 'no'. Of these twenty-five questions, twenty are related to neurotic symptoms, four to psychotic symptoms and one to convulsions. The SRQ-20 consists of the neurotic items only. These reflect depressive symptoms, anxiety, and psychosomatic complaints and have been found to detect probable cases of common mental disorder with reasonable accuracy [18].

The instrument met several criteria for use in this study, which was carried out by interviewers with 
limited training, within a limited period of time, among a large number of respondents, most of whom were illiterate. It is a self-report questionnaire; for illiterate respondents the questions may be read aloud by interviewers. Its administration time is $5-10$ minutes. The questions of the instrument are written in a simple, easy to understand language, and cover many important areas of psychopathology. The SRQ-20 has been used in many community-based surveys conducted in developing countries [11,19-21]. Additional to the widespread use of the 20 items version, the decision not to include items related to psychosis for the present study was also based on information from sociotherapy group facilitators: psychotic persons tended not to participate in the groups; not any psychotic participant was known of.

The SRQ-20 items are scored 0 ('no', symptom absent) or 1 ('yes', symptom present). Item scores are summarized to obtain a total score. A score above cut-off point indicates the existence of a probable menatl disorder. A cut-off score of 8 is widely used. However, optimal cutoff scores are shown to vary considerably across cultures, languages, settings, gender et cetera [21-28]. Factor structures of the SRQ-20 also vary across populations, ranging from two to seven $[21,29,30]$. Overall correspondence among the factor structures of the SRQ-20 is not found. So, at this stage the use of factor structures as subscales is not recommended. Besides, the variation in cut-off scores and factor structures emphasizes the need for performing separate validity studies among different populations.

\section{Instrument translation}

For this study, all SRQ-20 items were translated into Kinyarwanda by a bilingual Rwandan collaborator of the evaluation study (ThR), familiar with the intervention program and the (mental) health issues addressed by the instrument. Blind back-translation was done by another bilingual Rwandan, who was independent from, and not familiar with the intervention program or the study. This back-translation was examined by the first translator together with two other researchers (WFS, FV), which led to one minor change in the translation. Subsequently, the comprehensibility, acceptability, relevance and completeness of all items were discussed with the eight Rwandan interviewers working for the evaluation study. No changes were considered necessary [3].

\section{Study sample}

The SRQ-20 was administered to a sample of 418 adults (16 years and up); amongst these were 97 intervention (sociotherapy) group participants who had been selected randomly out of ten sociotherapy groups in or relatively near the study site (Byumba city) and yet correctly representing the intervention group with respect to gender and to urban or rural living situation; 92 relatives, friends or close collaborators of intervention group participants, i.e. one such person for every participant (these persons had each been randomly selected out of five persons listed by every group participant); and 229 randomly chosen other inhabitants of the district (the effectiveness study's control group). This sample will be referred to as the baseline sample (BA). Informed consent was obtained by use of an explanatory text, which because of the high illiteracy rate was read aloud. The interviewers were four men and four women who had been trained by two of the researchers (WFS, FV) during a three day training. They were all Rwandan sociology students at the 'Institut Polytechnique de Byumba'. They administered the questionnaire in a respectful way, not stopping respondents at 'yes' or 'no' and allowing more lengthy explanations.

A subsample of 99 was formed to establish the instrument's local validity and optimal cut-off point. This subsample will be referred to as the clinical interview (CI) sample. As no diagnostic or screening instrument with proven validity in a Rwandan context exists to be used as a 'gold standard', this subsample was clinically assessed by experienced clinicians. Meetings of intervention group participants and their selected relatives, friends or close collaborators were organized for administration of the SRQ-20. All respondents scoring 8 or above and an equal number scoring 7 or below, irrespective of whether they were intervention group participants or not, were also assessed by the clinicians, who were blind for the SRQ-20 scores. This procedure was repeated over similar meetings with different respondents untill 99 clinical assessments were completed.

Unfortunately, these assessments could not be done by trained clinicians from the same culture as the respondents. Rwanda harbours only three native psychiatrists, and these were not available for the study. Instead, the assessments were carried out by three of the Dutch researchers (WFS, FV, AvL) who are clinicians as well; all have broad experience in assessing psychopathology in non-western populations. They conducted a semistructured interview covering listed core symptoms of the disorders that were most likely to prevail in the specific context, i.e. depression, posttraumatic stress disorder, generalized anxiety disorder and panic disorder. It also contained questions to exclude psychosis and normal grief. If an assessor concluded that any of the mentioned disorders was present or possibly present in a subject, this subject was recorded as a 'case'. Interview agreement among the assessors was obtained by the following procedure: The assessors themselves designed the list of core symptoms as mentioned above. Next, two assessors witnessed an assessment carried out by the third one, after which all three independently made 
their own conclusions about 'caseness' of the respondent. There wasn't any verbal or non-verbal communication between the three clinicians untill all three had separately made and documented their diagnostic conclusions. The assessors took turns in taking the witnessing or the interviewing role. This procedure was followed sixteen times, fifteen times of which all three assessors drew identical conclusions. This resulted in $96 \%$ overall agreement and an interrater reliability (Fleiss' kappa) of 0.92. Since the assessors did not each by themselves conduct assessments of the same subjects, these agreement statistics might be inflated. The assessments were carried out with the aid of two of the intervention program's translators, familiar with western as well as local psychological idioms, and aware of the relevance of literal and neutral translation. The translators were also quite familiar with the clinicians, which allowed them to note and clarify possible (cross-cultural) misunderstandings between interviewers and respondents.

To assess the instrument's longitudinal validity a second, larger subsample (230 respondents) was re-assessed with the SRQ-20 by the same interviewers after a three months period, i.e. right after the intervention. This subsample will be referred to as the re-assessment (RA) sample. It was formed by all sociotherapy group participants and their relatives, friends or close collaborators who were available for both assessments, completed by a random selection of respondents from the control group matched on sex and age.

\section{Data analysis}

T-tests were conducted to compare mean scores of the SRQ-20. Internal reliability of the instrument was analyzed with Cronbach's alpha. Receiver operating characteristics (ROC) curves were used to explore the overall accuracy of the instrument to distinguish correctly between case and non-case, characterized by an area under the curve value (AUC). AUC values range from 0 to 1.0 , in which a value of 1.0 indicates a perfect prediction and a value of 0.5 indicates a prediction equal to chance. It was tested whether the criterion value of the SRQ-20 exceeded chance level (AUC > 0.5). Diagnostic sensitivity is the probability of a positive test result given the condition is present. Specificity is the probability of a negative test result given the condition is absent. Subsequently, a positive predictive value (PPV) is the probability of a positive diagnosis after a positive screening, and negative predictive value (NPV) is the probability of a negative diagnosis after a negative screening [31]. Predictive values range from 0 to 1 , in which a value closer to 1 reflects a better predictive value. Positive likelihood ratio (PLR) and negative likelihood ratio (NLR) provide direct estimates of an individual's chance of caseness. Likelihood ratios incorporate both sensitivity and specificity of the test. The PLR indicates how much the odds of the disease increase when a test is positive. The NLR indicates how much the odds of the disease decrease when a test is negative [32].

The degree of agreement between the results from the SRQ-20 and the clinical interviews is expressed both in percentage agreement and in Cohen's kappa coefficients. A kappa value of 1 reflects a perfect agreement between both observators, a kappa value of 0 reflects a degree of agreement as expected on base of chance. Kappa values in the range of $0.4-0.75$ can be interpreted as fair, kappa values exceeding 0.75 as good, and kappa values below 0.2 as slight agreement [33]. Diagnostic sensitivity and diagnostic specificity were plotted against each other to establish the optimal cut-off point. ROC-analysis and assessment of the psychometric qualities of the SRQ-20 were performed in the CI sample.

To test the longitudinal factorial invariance of the SRQ-20 we used exploratory factor analyses and confirmatory factor analyses. Product-moment correlation coefficients matrix was used to perform the factor analyses. We started with performing an exploratory factor analysis using principal axis factoring extraction in the BA sample to uncover the covariances between the twenty items of the SRQ-20. To facilitate interpretation varimax rotations were performed on the initial factor solutions. Based on the Kaiser-Guttman rule, factors with eigenvalues larger than 1 were retained for subsequent analyses. An item was assigned to a factor and used for factor labelling if its loading on that respective factor was larger than 0.35 and its loading to any other factor smaller than 0.35 [34]. Subsequently, confirmatory factor analyses were performed.

Confirmatory factor analysis (CFA) involves testing a series of hypothesized models relating to the instrument's measurement properties across samples. We started with testing the absolute fit of the factor structure in the BA and RA sample, consecutively. Subsequently, we used multigroup CFA to examine the extent of measurement invariance across these samples. In this analysis the fit between two hypothesized factor models is compared [35]. We distinguish the following models: 1) Model A: a model in which the number and pattern of factors are equal across samples; 2) Model B: model A with the additional constraint that factor loadings are equal across samples; 3) Model C: model B with the additional constraint that covariance matrices of factors are equal across samples; 4) Model D: model $\mathrm{C}$ with the additional constraint that error variances are equal across samples. Increment of fit between 1) model A and model $\mathrm{B}$; 2) model $\mathrm{B}$ and model $\mathrm{C}$; 3 ) model $\mathrm{C}$ and model $\mathrm{D}$ was tested using a $\chi^{2}$ test. If $\Delta \chi^{2}$ is not 
significant, the hypothesis of factorial invariance is tenable [36]. Sample sizes were adequate to test the fit of medium sized models [37]. Datanalyses were performed using PASW 17.0. Confirmative factor analyses were performed using Amos 16.0 [38].

\section{Results}

\section{Sociodemographic characteristics}

Male-female ratios were around 2:3 for the BA and CI samples and around 1:3 for the RA sample. Mean ages were around 35 years for the BA and CI samples and around 37 years for the RA sample. See Table 1.

\section{Predictive validity}

Reliability of the SRQ-20 over all samples is considered good (alphas ranging from 0.83 in CI sample to 0.87 in BA sample). Reliability in men and women, respectively, was also considered good (men: $\alpha=0.81$; women: $\alpha=$ 0.85 ). Mean total score of the SRQ-20 is 8.5 (S.D. = 3.5). Total scores showed no significant differences between men and women. Persons diagnosed having a mental disorder by the clinicians scored significantly ( $\mathrm{T}$ $(97)=4.325 ; \mathrm{p}<0.00)$ higher $($ mean $=11.3 ;$ S.D. $=4.1)$ compared to those having no mental disorder (mean = 7.3; S.D. $=4.2$ ). As no cases of psychosis were identified during clinical interviews, the existence of psychosis can be ruled out as a cause for disagreement between SRQ20 scores and clinical diagnoses.

The SRQ-20 performed moderately well in detecting common mental disorders. The AUC was 0.76. When analysed separately for men and women the SRQ-20 showed to perform equally well in men $(\mathrm{AUC}=0.74$ ) and women (AUC $=0.76$ ). See Figure 1 and Table 2 .

In evaluating the SRQ-20 as a potential screener for common mental disorder the most appropriate cut-off score is a trade-off between a high sensitivity and an acceptable specificity. In Rwanda, the SRQ-20 performs moderately well as a screener with a score of 10 as the optimal local cut-off point (sensitivity 0.69 ; specificity 0.79; see Table 3). The SRQ-20 performed better in women than in men. Cut-off scores differed also between men and women. The optimal cut-off point for men is 8 (sensitivity 0.69 ; specificity 0.65 ), while the optimal cutoff point for women is 10 (sensitivity 0.81 ; specificity 0.80 ). In evaluating likelihood ratios the optimal cut-off score combines the largest PLR with the smallest NLR. Both positive and negative predictive values and positive and negative likelihood ratios confirm the optimal cut-off scores. The PPV's of the cut-off scores can be considered good. The NPV's are relatively poor. This is consistent with the assumption that common mental disorder is prevalent in this traumatized society.

Cohen's kappa values of the SRQ-20 for the optimal cut-off scores were found fair in the total sample and among the women. The kappa value was poor to moderate for men.

\section{Exploratory factor analysis}

Principal factors extraction with varimax rotation was performed on all items of the SRQ-20 for the total sample $(n=418)$. Five factors were extracted, explaining $38 \%$ of the total variance. The number of items included for all five factors was 14. Six SRQ-20 items were not assigned to any factor, due to factor loadings $<0.35$ (items $4,5,14$ and 17) or factor loadings $>0.35$ on multiple factors (items 6 and 8). The factors reflected the following content: factor 1 : emotional and bodily symptoms of depression (items 1, 2, 3, 9 and 10); factor 2 : disability (items 11, 12 and 13); factor 3: digestive complaints (items 18 and 20); factor 4: lack of energy (items 7 and 19); and factor 5: loss of self esteem (items 15 and 16). Eigenvalues ranged from 5.80 to 1.02 .

\section{Confirmatory factor analysis}

To determine whether the SRQ-20 factor structure was invariant over time, single and multi sample

Table 1 Sociodemopgraphic characteristics of the three study samples

\begin{tabular}{|c|c|c|c|}
\hline & Baseline (BA) sample & Clinical Interview $(\mathrm{Cl})$ sample & Re-assessment (RA) sample \\
\hline Number of respondents & 418 & 99 & 230 \\
\hline \multicolumn{4}{|l|}{ Sex } \\
\hline Males, no (\%) & $163(39,0)$ & $42(42,4)$ & $55(23,9)^{*}$ \\
\hline Females, no (\%) & $255(61,0)$ & $57(57,6)$ & $175(76,1)$ \\
\hline Age (mean, S.D.) & $\begin{array}{c}16-87 \\
(\mu=35,3 ; \text { S.D. }=15,0)\end{array}$ & $\begin{array}{c}16-74 \\
(\mu=34,7 ; \text { S.D. }=14,1)\end{array}$ & $\begin{array}{c}16-77 \\
(\mu=37,2 ; \text { S.D. }=13,9)^{*}\end{array}$ \\
\hline \multicolumn{4}{|l|}{ Treatment condition ${ }^{1}$} \\
\hline Direct, no (\%) & $97(54,8)$ & $52(52,5)^{*}$ & $90(39,1)^{*}$ \\
\hline Indirect, no (\%) & $92(23,2)$ & $47(47,5)$ & $77(33,5)$ \\
\hline Control, no (\%) & $229(22,0)$ & - & $63(27,4)$ \\
\hline
\end{tabular}

* $P<0.05$, means and proportions tested against the total sample

${ }^{1}$ direct = sociotherapy group participants; indirect = relatives, friends or close collaborators of sociotherapy group participants; control = random community sample. 


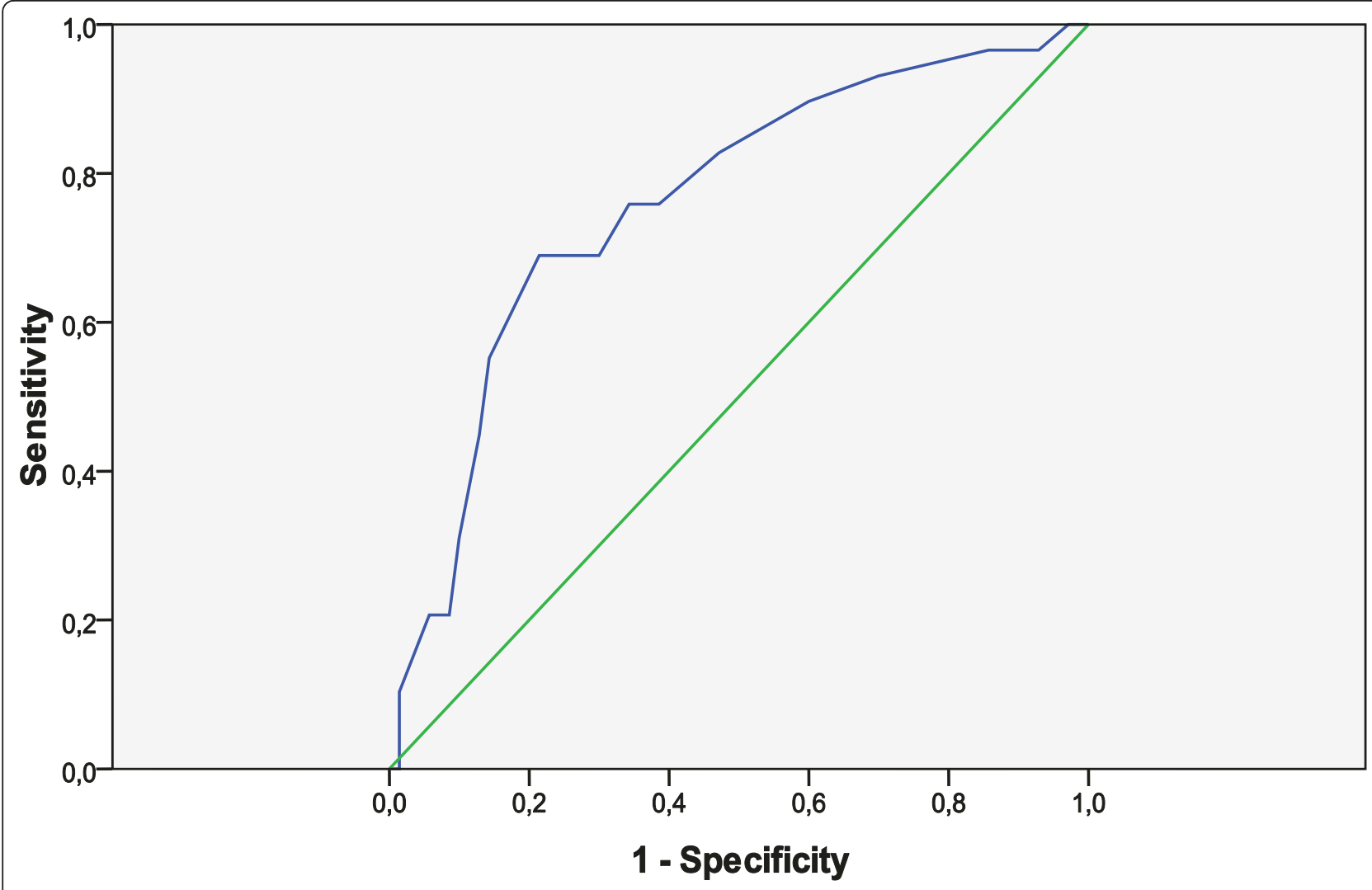

\section{Diagonal segments are produced by ties.}

Figure 1 Receiving Operator Characteristic (ROC) curve of the SRQ-20 scores in the presence or absence of caseness as diagnosed by the clinicians.

confirmatory factor analysis was performed on the five factors of the SRQ-20. The five factors were hypothesized to covary with one another.

The assumption of normality was evaluated through AMOS 16.0. The RA sample showed significant skewness. Mardia's coefficient for multivariate kutosis was 9,289 in the BA sample and 17,122 in the RA sample, indicating a non-normal multivariate distribution of the data. No outliers were observed (using Mahalanobis distance). CFA was performed using data from the BA sample $(n=418)$ and RA sample $(n=230)$. There were no missing data.

Table 2 SRQ-20: Area under the curve, lower and upper limit

\begin{tabular}{cccccc}
\hline & AUC & LL & UL & Z-value & $P$-value \\
\hline Male & 0.74 & 0.59 & 0.90 & 3.077 & 0.013 \\
Female & 0.76 & 0.62 & 0.91 & 3.467 & 0.002 \\
Total & 0.76 & 0.66 & 0.86 & 4.815 & $<0.001$ \\
\hline
\end{tabular}

We identified the fit of the hypothesized model in the single samples. In the BA sample the hypothesized model showed good fit with the data, where $\chi^{2}(67)=$ 93.243, RMSEA $=0.031$, $\mathrm{RMR}=0.008, \mathrm{GFI}=0.97$, CFI $=0.98$ and TLI $=0.97$. In the RA sample the model showed excellent fit with the data, where $\chi^{2}(67)=$ 70.001, RMSEA $=0.014, \mathrm{RMR}=0.008$, GFI $=0.96$, CFI $=1.00$ and TLI $=0.99$. Subsequently we conducted $\mathrm{a}$ multisample CFA. Maximum likelihood estimation was employed to test the fit of all models. The hypothesized model fitted the data well $\left(\chi^{2}(134)=163.27, P=0.043\right)$. A follow-up Bollen-Stine bootstrapped analysis was performed with 200 replications to correct for non-normality of the data. This resulted in better $\chi^{2}$ and $P$ values, $\chi^{2}=146.36$ and $P=0.214$.

No significant differences were found between the unconstrained model and the models with constrained factors, factor loadings and covariances. There was, however, a significant difference between model $\mathrm{C}$ and model $\mathrm{D}$, indicating a longitudinal invariance on the 
Table 3 Psychometric properties of the SRQ-20 with different cut-off scores

\begin{tabular}{|c|c|c|c|c|c|c|c|c|c|}
\hline & Cut-off & Sensitivity & Specificity & PPV & NPV & PLR & NLR & Agreement & kappa \\
\hline \multirow[t]{3}{*}{ Male } & 7 & 0.69 & 0.59 & 0.81 & 0.43 & 1.67 & 0.52 & $62 \%$ & 0.24 \\
\hline & $8^{*}$ & 0.69 & 0.65 & 0.83 & 0.47 & 2.01 & 0.47 & $67 \%$ & 0.31 \\
\hline & 9 & 0.54 & 0.69 & 0.77 & 0.44 & 1.74 & 0.67 & $64 \%$ & 0.21 \\
\hline \multirow[t]{4}{*}{ Female } & 8 & 0.81 & 0.66 & 0.90 & 0.48 & 2.38 & 0.28 & $70 \%$ & 0.39 \\
\hline & 9 & 0.81 & 0.71 & 0.91 & 0.52 & 2.78 & 0.27 & $74 \%$ & 0.44 \\
\hline & $10^{*}$ & 0.81 & 0.80 & 0.92 & 0.62 & 4.16 & 0.23 & $81 \%$ & 0.56 \\
\hline & 11 & 0.69 & 0.83 & 0.87 & 0.61 & 4.03 & 0.38 & $79 \%$ & 0.50 \\
\hline \multirow[t]{5}{*}{ Total } & 7 & 0.76 & 0.61 & 0.86 & 0.45 & 1.97 & 0.39 & $66 \%$ & 0.31 \\
\hline & 8 & 0.76 & 0.66 & 0.87 & 0.48 & 2.12 & 0.37 & $69 \%$ & 0.36 \\
\hline & 9 & 0.69 & 0.70 & 0.84 & 0.49 & 2.30 & 0.44 & $70 \%$ & 0.35 \\
\hline & $10^{*}$ & 0.69 & 0.79 & 0.86 & 0.57 & 3.22 & 0.39 & $76 \%$ & 0.45 \\
\hline & 11 & 0.55 & 0.86 & 0.82 & 0.62 & 3.86 & 0.52 & $77 \%$ & 0.42 \\
\hline
\end{tabular}

* Optimal cut-of score; scores above this cut-off value give the best indication for possible psychopathology.

Note: PPV: positive predictive value; NPV: negative predictive value; PLR: positive likelihood ratio; NLR: negative likelihood ratio.

residual level. See Table 4 for detailed multigroup comparison fit indices.

\section{Discussion}

This study shows the SRQ-20 can be used as a screener to detect mental disorder in a Rwandan community sample. However, cut-off scores need to be adjusted. Rwandan women and men have different optimal cutoff scores. Among men a cut-off score of 8 was optimal, among women a cut-off score of 10 was optimal. Differences between men and women were also found in a validation study among a traumatized population in Eastern Afghanistan [21]. Compared with other traumatized populations, the cut-off in the Rwandan population is relatively low, suggesting a more introvert expression of psychological distress.

In the present study the SRQ-20 performed less well in males than in females. This may be due to the country's atmosphere, which is still paranoid after the mass violence that took place. Especially men show a tendency not to trust others easily and to keep problems inside. Qualitative information consistently points out that men in Rwanda generally do not share emotional problems. This may have impacted the intervention's effect on men as well as the validity of data collected from male respondents. It may also explain the difference in optimal cut-off points between men and women.

A comparable problem may apply to ethnic background. Given the country's recent history this is an extremely sensitive issue, not to be addressed by interviewers during a brief one-time meeting. Besides, many residents have a mixed Hutu-Tutsi background - the distinction which is usually made. Yet, one can not rule out the possibility that ethnic background impacted the way people responded to the intervention as well as to certain questions of the interview, and therefore acts as an independent variable. The same goes for age, as age impacts the mental health consequences of past experiences. Our study samples represent all age groups, and therefore our findings only apply to the use of the SRQ20 in random community samples.

For the present study we used a stratified sample to ensure sufficient variance in our SRQ scores. The actual prevalance of psychopathology in the Rwandan community is unknown and, as a result, we were not able to weigh the sample accordingly in our analyses. This is an

Table 4 Results of multigroup confirmative factor analysis using data from BA sample $(n=418)$ and RA sample $(n=$ 230)

\begin{tabular}{|c|c|c|c|c|c|c|c|c|c|}
\hline & $x^{2}$ & $\Delta \chi^{2}$ & $\Delta \mathrm{df}$ & $P$ & RMSEA $(90 \% \mathrm{Cl})$ & RMR & GFI & CFI & TLI \\
\hline $\begin{array}{l}\text { Model A: } \\
\text { Equal factors }\end{array}$ & 163.27 & - & - & - & $0.018(0.003-0.028)$ & 0.008 & 0.97 & 0.99 & 0.98 \\
\hline $\begin{array}{l}\text { Model B: } \\
\text { Equal factor loadings }\end{array}$ & 165.76 & 2.50 & 9 & 0.98 & $0.016(0.000-0.025)$ & 0.008 & 0.97 & 0.99 & 0.99 \\
\hline $\begin{array}{l}\text { Model C: } \\
\text { Equal covariances }\end{array}$ & 190.29 & 24.53 & 15 & 0.06 & $0.018(0.004-0.026)$ & 0.012 & 0.96 & 0.98 & 0.98 \\
\hline $\begin{array}{l}\text { Model D: } \\
\text { Equal error variances }\end{array}$ & 269.23 & 78.94 & 14 & 0.000 & $0.030(0.023-0.036)$ & 0.013 & 0.95 & 0.95 & 0.95 \\
\hline
\end{tabular}

Note: $\Delta \chi^{2}:$ nested $\chi^{2}$ difference; $\Delta \mathrm{df}$ : nested df difference; $P$ : $P$-value assuming the less restrained model to be correct; RMSEA: root mean square error of approximation; $90 \%$ Cl: $90 \%$ confidence interval; RMR: root mean square residual; GFI: goodness of fit index; CFI: comparative fit index; TLI: Tuckler-Lewis Index. 
important limitation of our study. Misrepresentation of the prevalence rate does not affect PPV and NPV estimations, but it does bias sensitivity, specificity, PLR, NLR, agreement and kappa estimations. However, the extent of this bias is unknown. Several studies in war affected populations and particularly in Rwanda found extremely high rates of depression, PTSD and other anxiety disorders [39-44]. The prevalence in our sample was $69 \%$. It should be noted that we did not use diagnostic instruments but a screener, thereby identifying possible cases and capturing a variety of possible diagnoses in one measure. Therefore, a very high prevalence could be expected in our sample, quite possibly representing the actual prevalence. Future research is needed to confirm the sensitivity and specificity estimates of the SRQ-20 in a Rwandan community sample.

When dichotomous items are concerned, ideally a tetrachoric matrix should form the input of the exploratory factor analysis and confirmatory factor analyses [45]. In our case, the resulting tetrachoric matrix calculated using SPSS-Macro TetCorr Version 2.3 [46] from our data was non-positive definite, which made it impossible to compute a factor solution using principal axis factoring. This is a common problem in analysing symptom data, often caused by large correlations, insufficient sample size, or a non-normal underlying distribution $[45,47]$. Principal component analysis is less sensitive to these issues, but since our purpose was to structure the correlations among our variables, e.g., to explore a parsimonious representation among our measured variables instead of reducing the number of items, we decided against it. Also, the bias caused by using this method of analysis is less important when exploring the clustering of the items is the main purpose of the analysis, as it was in our case. All things considered, we chose to employ a less sophisticated method of analysis, i.c. employing a Pearsons correlation matrix as basis for our factor analysis. Previous studies on the factor structure of the SRQ also used a Person correlation matrix as basis of their analyses $[21,29,30]$.

Since the factor structure of the SRQ-20 in this study proves to be time invariant, the instrument meets an important criterion to measure symptom change over time [17]. The results show that the number of factors, factor loadings and covariances of the factors remain equal over time. Only at residual level time invariances exist. This residual invariance might account for the differences in factor structures found across cultures. It is, however, important to realise that differences exist between our BA and RA samples, which may have caused residual invariance. Also, residual variances are reflective of individual variances in response to factors such as the intervention [48]. That is, at pretest the variances will be more homogeneous because individuals are more similar with regard to their level of psychological complaints. At posttest individual differences will be more pronounced because some participants respond favourably to the intervention, whereas others do not. Furthermore, in our samples data were not multivariate normally distributed. It is known that non-normality inflates the $\chi^{2}$-statistic of overall model fit, thereby increasing the chance of type 1 errors, i.e. the chance to abusively reject a hypothesized model [49]. Overall, methodologists agree that the test of equal residual variances is highly stringent and will rarely hold in realistic datasets [50]. Residual invariance is therefore not as important to the evaluation of measurement invariance as the test of equal form and factor loadings.

The data in our samples were categorical and nonnormal in nature. Maximum likelihood estimation (MLE) of models with this type of data is not recommended. Asymptotic Distribution Free (ADF) or robust weighted least square estimator (WLSMW) might be a more appropriate choice. Unfortunately, the use of ADF requires sample sizes that exceed 1000 cases and small models. So, while ADF analysis may be theoretically optimal, it is not a practical method [51]. Also, WLSMW is not offered in AMOS 16.0. Since MLE is less problematic when analyzing the covariance matrix, and since Fouladi [52] found in a simulation study that the Bollen-Stine test of overall model fit performed well relative to other methods of testing model fit, we decided to estimate the model with MLE and additional Bollen-Stine bootstrapping.

The factor structure of the SRQ-20 in this study differed from factor structures reported in literature. Even in comparable settings numbers and contents of factors showed differences. For instance, in a primary care sample in an Eastern Afghanistan post-conflict setting, the SRQ-20 resulted in two factors, namely 'common disorder' and 'social disability' [21]. This study further emphasizes the need to establish the optimal cut-off scores for each setting, and not to use factors as separate subscales.

Lastly, this study confronted the researchers with various cross-cultural challenges. Extensive qualitative research and ample time and human resources are required to optimally deal with issues like the local validity of items of the SRQ-20, limited response options ('yes' or 'no'), and culturally sensitive clinical assessment. Our limited resources to tackle these issues may have impacted the cross-cultural validity of our findings.

\section{Conclusions}

The SRQ-20 can be used as a screener to detect mental disorder in a Rwandan community sample. However, cut-off scores need to be adjusted. In this study setting, the instrument also shows longitudinal factorial 
invariance, which is an important prerequisite for assessing changes in symptom severity. This is a significant finding as in non-western post-conflict settings the relevance of diagnostic categories is questionable. The use of the SRQ-20 can be considered an alternative option for measuring the effect of a psychosocial intervention on mental health.

\section{Acknowledgements}

This study was supported by a grant from the Health Research Development Counsel, Department Prevention Program (ZonMW), OOG-Geestkracht (ZonMW: 60-60105-98-117), and by grants form Cordaid (The Hague) and Prins Bernhard Cultuurfonds (Amsterdam). Other than independent reviewers' comments on the initial proposal, these three funding bodies of the study had no further role in the study design, data collection, data analysis, data interpretation, writing, or the decision to submit the report for publication.

We thank Ngendahayo Emmanuel and Sarabwe Emmanuel from the Episcopal Church in Byumba, Rwanda, for helping us organise and conduct the research. We also thank our loyal interviewers: Rukundo Ange, MugishaBitanuzire John-Peter, Umuhoza Adelin, Uwizeye Beata, Ingabire Bernadette, Uwizeyimana Albertine, Mukanyiligira Marie Grace, and Muhongwanseko Scholastique.

\section{Author details}

'Department of Psychiatry, Academic Medical Center, University of Amsterdam, Netherlands, Meibergdreef 5, 1105 AZ Amsterdam, Netherlands. ${ }^{2}$ Equator Foundation, Netherlands, Nienoord 5, 1112 XE Diemen, Netherlands. ${ }^{3}$ Episcopal Church of Rwanda, Diocese of Byumba, Rwanda, P.O. Box 17, Byumba, Rwanda. ${ }^{4}$ Department of Psychiatry, O3 Mental Health Care Research Center, Erasmus MC, Rotterdam, Netherlands, 's-Gravendijkwal 230, 3015 CE Rotterdam, Netherlands.

\section{Authors' contributions}

WFS participated in conceiving and designing the study and in the data collection, and in drafting and editing the manuscript. FV participated in conceiving and designing the study and in the data collection, and in editing the manuscript. AvL participated in the data collection and in editing the manuscript. TR participated in the data collection and editing the manuscript. AMK participated in conceiving and designing the study, performed the statistical analysis and participated in drafting and editing the manuscript. All authors read and approved the final manuscript.

\section{Competing interests}

The authors declare that they have no competing interests.

Received: 22 November 2010 Accepted: 16 August 2011 Published: 16 August 2011

\section{References}

1. Richters A, Dekker C, Scholte WF: Community based sociotherapy in Byumba, Rwanda. Intervention 2008, 6:100-116.

2. Richters $\mathrm{A}$, Rutayisire $\mathrm{T}$, Dekker $\mathrm{C}$ : Care as a turning point in sociotherapy: Remakig the moral world in post-genocide Rwanda. Medische Antropologie 2010, 22:93-108.

3. Verduin F, Scholte WF, Rutayisire T, Richters A: How qualitative information helped to shape quantitative research instruments in Rwanda. Intervention 2010, 8:233-244.

4. American Psychiatric Association: Diagnostic and statistical manual of mental disorders DSM-IV-TR. 4 edition. Arlington, VA; 2000.

5. World Health Organization: The ICD-10 classification of mental and behavioural disorders: clinical descriptions and diagnostic quidelines Geneva; 1992.

6. Ager A: Psychosocial needs in complex emergencies. Lancet 2002, 360:43-44.

7. Weiss M, Saraceno B, Saxena S, van Ommeren M: Mental health in the aftermath of disasters: consensus and controversy. J Nerv Ment Dis 2003, 191:611-615.
8. Miller K, Kulkarni M, Kushner H: Beyond trauma-focused psychiatric epidemiology: bridging research and practice with war-affected populations. Am J Orthopsychiatry 2006, 76:409-422.

9. Bolton P, Stichick Betancourt P: Mental health in post-war Afghanistan. JAMA 2004, 292:626-628.

10. Miller KE, Rasmussen A: War exposure, daily stressors, and mental health in conflict and post-conflict settings: bridging the divide between trauma-focused and psychosocial frameworks. Soc Sci Med 2010, 70:7-16.

11. Harding TW, Arango MV, Baltazar J, Climent CE, Ibrahim HHA, LadridoIgnacio L, Srinivasa Murthy R, Wig NN: Mental disorders in primary health care: a study of the frequency and diagnosis in four developing countries. Psychol Med 1980, 10:231-242.

12. Beusenberg M, Orley J: A user's quide to the Self Reporting Questionnaire (SRQ) Geneva: World Health Organization; 1994.

13. Van Ommeren M, Thapa S, Makaju R, Prasain D, Bhattarai R, de Jong J: Preparing instruments for transcultural research: use of the translation monitoring form with Nepali-speaking Bhutanese refugees. Transcult Psychiatry 1999, 36:285-301

14. Manson SM: Cross-cultural and multi-ethnic assessment of trauma. In Assessing psychological trauma and PTSD: A handbook for practitioners. Edited by: Wilson JP, Keane TM. New York: Guilford Press; 1997:239-266.

15. Flaherty JA, Gavira FM, Pathak D, Mitchell T, Wintrob R, Richman JA, Birz S: Developing instruments for cross-cultural psychiatric research. J Nerv Ment Dis 1988, 176:257-263.

16. Neuner F, Schauer M, Klaschik C, Karunakara U, Elbert T: A comparison of Narrative Exposure Therapy, supportive counseling, and psychoeducation for treating posttraumatic stress disorder in an African refugee settlement. J Consult Clin Psychol 2004, 72:579-587.

17. Vandenberg RJ, Lance CE: A review and synthesis of the measurement invariance literature: Suggestions, practices, and recommendations for organizational research. Organizational Research Methods 2000, 3:4-69.

18. Sartorius N, Janca A: Psychiatric assessment instruments developed by the World Health Organization. Soc Psychiatry Psychiatr Epidemiol 1996, 31:55-69.

19. Rumble S, Swartz L, Parry C, Zwarenstein M: Prevalence of psychiatric morbidity in the adult population of a rural South African village. Psychol Med 1996, 26:997-1007.

20. Bhagwanjee A, Parekh A, Paruk Z, Petersen I, Subedar H: Prevalence of minor psychiatric disorders in an adult African rural community in South Africa. Psychol Med 1998, 28:1137-1147.

21. Ventevogel P, de Vries G, Scholte WF, Rasa Shinwari N, Faiz H, Nassery R, van den Brink W, Olff M: Properties of the Hopkins Symptom Checklist-25 (HSCL-25) and the Self-Reporting Questionnaire (SRQ-20) as screening instruments used in primary care in Afghanistan. Soc Psychiatry Psychiat Epidemiol 2007, 42:328-335.

22. Mari JJ, Williams P: A validity study of a psychiatric screening Questionnaire (SRQ-20) in primary care in the city of Sao Paulo. Br J Psychiatry 1986, 148:23-26.

23. Upadhyaya A, Creed F, Upadhyaya M: Psychiatric morbidity among mothers attending well-baby clinic: a cross-cultural comparison. Acta Psychiatr Scand 1989, 81:148-151.

24. Kortmann F: Psychiatric case finding in Ethiopia: shortcomings of the Self-Reporting Questionnaire. Cult Med Psychiatry 1990, 14:381-391.

25. Araya R, Wynn R, Lewis $\mathrm{G}$ : Comparison of two self-administered psychiatric questionnaires (GHQ-12 and SRQ-20) in primary care in Chile. Soc Psychiatry Psychiatr Epidemiol 1992, 27:168-173.

26. Ghubash R, Daradkeh T, El-Rufaie OF, Abou-Saleh MT: A comparison of the validity of two psychiatric screening questionnaires: the Arabic General Health Questionnaire (AGHQ) and Self-Reporting Questionnaire (SRQ-20) in UAE, using receiver operating characteristics (ROC) analysis. Eur Psychiatry 2001, 16:122-126.

27. Pollock Jl, Maneski-Holland S, Patel V: Detection of depression in women of child-bearing age in non-western cultures: a comparison of the Edinburgh Postnatal Depression Scale and the Self-Reporting Questionnaire-20 in Mongolia. J Affective Dis 2006, 92:267-271.

28. Giang KB, Allebeck P, Kullgren G, Tuan NV: The Vietnamese version of the Self-Reporting Questionnaire 20 (SRQ-20) in detecting mental disorders in rural Vietnam: a validation study. Int I Soc Psychiatry 2006, 52:175-184.

29. lacoponi $E$, Mari JJ: Reliability and factor structure of the Portuguese version of Self-Reporting Questionnaire. Int J Soc Psychiatry 1989, 35:213-222. 
30. Cherian VI, Peltzer K, Cherian L: The factor structure of the Self-Reporting Questionnaire (SRQ-20) in South Africa. East African Med J 1998, 75:654-656.

31. Altman DG, Bland JM: Statistics notes: diagnostic tests 2: predictive values. BMJ 1994, 309:102.

32. Attia J: Moving beyond sensitivity and specificity: using likelihood ratios to help interpet diagnostic tests. Australian Prescriber 2003, 26:111-113.

33. Landis JR, Koch GG: The measurement of observer agreement for categorical data. Biometrics 1977, 33:159-174

34. Nunally JC: Psychometric theory. 2 edition. New York: McGraw-Hill; 1978.

35. Byrne BM, Shavelson RJ, Muthen B: Testing for the equivalence of factor covariance and mean structures: the issue of partial measurement invariance. Psychol Bull 1989, 105:456-466.

36. Tabachnick BG, Fidell LS: Using multivariate statistics. 4 edition. Needham Heights, MA: Allyn \& Bacon; 1996.

37. Boomsma A: On the robustness of LISREL (maximum likelihood estimation) against small sample size and nonnormality. PhD thesis University of Groningen; 1983.

38. Arbuckle JL: Amos 16.0 user's guide Spring House, PA: Amos Development Corporation; 1995.

39. De Jong JTVM, Komproe $\mathrm{H}$ : Closing the gap between psychiatric epidemiology and mental health in postconflict situations. Lancet 2002, 359:1793-1794.

40. Rodin D, Van Ommeren M: Explaining enormous variations in rates of disorder in trauma-focused psychiatric epidemiology after major emergencies. Int J Epidemiol 2009, 38:1045-1048.

41. Neugebauer R, Fisher PW, Turner JB, Yamabe S, Sarsfield JA, Stehling-Arisa T: Post-traumatic stress reactions among Rwandan children and adolescents in the early aftermath of genocide. Int J Epidemiol 2009, 38:1033-1045

42. Sezibera V, Van Broeck N, Philippot P: Intervening on persistent posttraumatic stress disorder: Rumination-focused cognitive and behavioral therapy in a population of young survivors of the 1994 genocide in Rwanda. J Cognitive Psychother 2009, 23:107-113.

43. Brounéus $K$ : The trauma of truth telling: Effects of witnessing in the Rwandan Gacaca courts on psychological health. J Conflict Resolution 2010, 54:408-437.

44. Hagengimana A, Hinton D, Bird B, Pollack M, Pitman RK: Somatic panicattack equivalents in a community sample of Rwandan widows who survived the 1994 genocide. Psychiatry Res 2003, 117:1-9.

45. Muthen B, Hofacker Ch: Testing the assumptions underlying tetrachoric correlations. Psychometrika 1988, 53:563-577.

46. Enzmann D: Tetcorr version 2.3. 2007 [http://www2.jura.uni-hamburg.de/ instkrim/kriminologie/Mitarbeiter/Enzmann/Software/Enzmann_Software_. html].

47. Muthen B: Dichotomous factor analysis on symptom data. Sociological Meth Res 1989, 18:19-65.

48. Brown TA: Confirmatory factor analysis for applied research New York: Guilford Press; 2006

49. West S, Finch JF, Curran PJ: Structural Equation Models with nonnormal variables: problems and remedies. In Structural Equation Modelling: concepts, issues, and applications. Edited by: Hoyle RH. Thousand Oaks, CA Sage; 1995:56-75.

50. Chan D: The conceptualization and analysis of change over time: an integrative approach incorporating longitudinal and covariance structures analysis (LMACS) and multiple indicator growth modelling (MLGM). Organizational Research Methods 1998, 1:421-483.

51. Muthen BO: Goodness of fit with categorical and other nonnormal variables. In Testing Structural Equation Models. Edited by: Bollen KA, Long JS. Newbury Park, CA: Sage; 1993:205-243.

52. Fouladi RT: Covariance structure analysis techniques under conditions of multivariate normality and nonnormality - Modified and bootstrap test statistics. Proceedings of the Annual Meeting of the American Educational Research Association San Diego, CA; 1998.

\section{Pre-publication history}

The pre-publication history for this paper can be accessed here:

http://www.biomedcentral.com/1471-2288/11/116/prepub doi:10.1186/1471-2288-11-116

Cite this article as: Scholte et al:: Psychometric properties and longitudinal validation of the self-reporting questionnaire (SRQ-20) in a Rwandan community setting: a validation study. BMC Medical Research Methodology 2011 11:116.

\section{Submit your next manuscript to BioMed Central and take full advantage of:}

- Convenient online submission

- Thorough peer review

- No space constraints or color figure charges

- Immediate publication on acceptance

- Inclusion in PubMed, CAS, Scopus and Google Scholar

- Research which is freely available for redistribution 